\title{
Inflammatory cytokines, appetite-regulating hormones, and energy metabolism in patients with gastrointestinal cancer
}

\author{
AYAKA SHINSYU ${ }^{1}$, SHIGEKI BAMBA ${ }^{1}$, MIKA KURIHARA ${ }^{1}$, HIROSHI MATSUMOTO ${ }^{2}$, \\ AYANO SONODA $^{2}$, OSAMU INATOMI ${ }^{2}$, AKIRA ANDOH $^{2}$, KATSUSHI TAKEBAYASHI $^{3}$, \\ MASATSUGU KOJIMA ${ }^{3}$, HIROYA IIDA ${ }^{3}$, MASAJI TANI $^{3}$ and MASAYA SASAKI ${ }^{1}$ \\ Divisions of ${ }^{1}$ Clinical Nutrition, ${ }^{2}$ Gastroenterology and ${ }^{3}$ Gastrointestinal Surgery, \\ Shiga University of Medical Science, Otsu, Shiga 520-2192, Japan
}

Received September 20, 2019; Accepted December 17, 2019

DOI: 10.3892/ol.2020.11662

\begin{abstract}
This study investigated energy metabolism and its association with inflammatory cytokines and appetiteregulating hormones in patients with gastrointestinal cancer. Subjects were inpatients scheduled to undergo therapeutic intervention for diagnosed gastrointestinal cancer. Nutritional status on admission was assessed based on anthropometric measurements, nutrition screening results, food intake rate (energy intake/energy provided in hospital food), and biochemical test results. Fat-free mass (FFM) was measured using the bioelectrical impedance analysis. Resting energy expenditure (REE) and respiratory quotient were measured with indirect calorimetry, and basal energy expenditure (BEE) was calculated using the Harris-Benedict equation. A total 51 patients with gastrointestinal cancer were enrolled (17 with esophageal cancer, 15 with gastric cancer, and 19 with colorectal cancer); 16 had stage I disease, 11 had stage II, 13 had stage III, and 11 had stage IV. The levels of inflammatory cytokines such as interleukin (IL)-6 and tumor necrosis factor (TNF)- $\alpha$ increased significantly with cancer stage progression $(\mathrm{P}<0.001$; Jonckheere-Terpstra trend test). The REE/body weight and the $\mathrm{REE} / \mathrm{FFM}$ tended to increase with cancer stage progression $(\mathrm{P}=0.064$ and $\mathrm{P}=0.053$, respectively; Jonckheere-Terpstra trend test). FFM showed a significant negative correlation with the level of TNF- $\alpha(\mathrm{P}=0.008$; Spearman's correlation coefficient). Also, food intake rate showed a significant negative correlation with levels of IL- 6 and TNF- $\alpha(\mathrm{P}<0.001)$. The level of active ghrelin was positively correlated with that of IL-6 and energy metabolism ( $\mathrm{P}=0.004$ and 0.016 , respectively) and negatively correlated with food intake rate $(\mathrm{P}=0.035)$, which suggests a state of ghrelin resistance. In conclusion, this study
\end{abstract}

Correspondence to: Dr Shigeki Bamba, Division of Clinical Nutrition, Shiga University of Medical Science, Seta-Tsukinowa, Otsu, Shiga 520-2192, Japan

E-mail: sb@belle.shiga-med.ac.jp

Key words: indirect calorimetry, esophageal cancer, gastric cancer, colon cancer confirmed increases in the levels of inflammatory cytokines with the progression of gastrointestinal cancer and suggested the possible association of such increases with decreased FFM and the increased energy metabolism. However, the increased levels of active ghrelin failed to compensate for cachexia in cancer patients.

\section{Introduction}

Malnutrition occurs in $20-70 \%$ of cancer patients, and patients with gastrointestinal cancer are at particularly high risk (1). Also, malnutrition is reported to occur more frequently with cancer progression (1). According to a study by Zhang et al, only $2 \%$ of patients with advanced gastrointestinal cancer did not require nutrition intervention, and $57.4 \%$ of them required management of malnutrition-related symptoms and nutritional support (2).

Proinflammatory cytokines such as interleukin (IL)-6 and tumor necrosis factor (TNF)- $\alpha$ are known to have the pro-tumorgenic functions (3) and are also produced by cancer tissues, including interstitial cells (4). The serum levels of these cytokines were reported to positively correlate with cancer stages (4-7). These cytokines can affect neuroendocrine control of appetite, leading to anorexia and hypermetabolism, resulting in muscle wasting (1). Cancer-induced metabolic disorder progresses gradually, eventually leading to refractory cachexia (8). Inflammatory cytokines stimulate the activity of anorectic proopiomelanocortin neurons and inhibit the activity of orexigenic neuropeptide Y (NPY) neurons in patients with cachexia (9). Inflammatory cytokines also induce NF- $\kappa \mathrm{B}$ activation. $N F-\kappa B$ affects the expression of genes that regulate the ubiquitin proteasome pathway (UPP) and promotes the loss of protein (10), resulting in decreased in fat-free mass (FFM).

Appetite-regulating hormones such as ghrelin and leptin, play an important role in cancer patients. Ghrelin is present in two forms: An inactive form known as deacylated ghrelin, and an active form, the acylated ghrelin that accounts for $\sim 10 \%$ of the total amount of ghrelin and is synthesized under the action of ghrelin O-acyltransferase (GOAT) (11-13). GOAT expression and activity are modulated by nutrient availability, particularly by the availability of medium-chain fatty acids, which are used as acylation substrates and promote acyl-ghrelin production 
and secretion (14). Ghrelin is secreted from the stomach and acts on the hypothalamic NPY, an appetite promoting peptide, to increase appetite and suppress energy metabolism $(11,15,16)$, whereas leptin is secreted from adipocytes and acts on the hypothalamus to suppress food intake and increase energy metabolism (15). Total ghrelin levels were found to be significantly higher in cancer patients with cachexia than in cancer patients without cachexia (17-19), which suggests that cachexia could be a state of ghrelin resistance accompanied by increases in active ghrelin and the ratio of acylated to total ghrelin levels (20). However, the precise mechanism of ghrelin resistance is unknown. It is also possible that ghrelin levels increase to compensate for the increased metabolic rate and energy often observed in patients with cancer cachexia (21).

Early detection and assessment of weight loss and undernutrition, as well as provision of adequate nutrition therapy, enable cancer patients to maintain good nutritional status (1). In this study, we focused on the following two screening tools for assessment of nutritional status: The Subjective Global Assessment (SGA), described by Baker et al in 1982 (22), which assesses nutritional status based solely on disease history and findings of physical examination; and the Patient-Generated Subjective Global Assessment (PG-SGA), which was proposed by Ottery in 1994 and has been used by the American Dietetic Association as a screening tool for cancer patients (23). The PG-SGA comprises items included in the SGA as well as items to assess problems affecting dietary intake and nutritional status in cancer patients. Bauer et al reported that the undernourished status in cancer patients can be assessed in the early stages using PG-SGA (24).

There is no well-accepted concept of energy metabolism in patients with gastrointestinal cancer. Some reports have found that resting energy expenditure (REE) and basal energy expenditure (BEE) were similar in cancer patients $(25,26)$, while others have found that REE was greater than BEE in cancer (27-30). Although increases in REE with cancer stage progression were shown in one study (31), the differences in nutritional status and energy metabolism by different cancer locations were not well investigated.

This study examined nutritional status in patients with gastrointestinal cancer by cancer stage and also by cancer location in order to investigate factors influencing REE. The effect of inflammatory cytokines (IL-6 and TNF- $\alpha$ ) and appetite-regulating hormones (ghrelin and leptin) on FFM and energy metabolism were also investigated.

\section{Patients and methods}

Patients. Subjects were patients aged $<80$ years who were admitted to Shiga University of Medical Science Hospital for treatment (surgery, chemotherapy, and radiotherapy) following a diagnosis of gastrointestinal cancer (esophageal cancer, gastric cancer, or colorectal cancer) between June 2014 and October 2018. To eliminate the influence of prior treatment as much as possible, the included patients were those who had not undergone the above types of cancer treatment previously and those who had received the latest dose of chemotherapy or radiotherapy $\geq 1$ month before admission and had no adverse reactions to prior treatment. Exclusion criteria were age $<20$ years or $\geq 80$ years; physician-diagnosed refractory cachexia; severe obesity [body mass index (BMI)] $\geq 30 \mathrm{~kg} / \mathrm{m}^{2}$ ), hyper- or hypo-metabolic conditions (e.g., thyroid disorders, liver cirrhosis, pulmonary disease, cardiac failure, and Wernicke's encephalopathy), and dialysis. The Union for International Cancer Control (UICC) classification system was used for staging of gastrointestinal cancer. The present study was conducted with approval by The Ethics Committee of Shiga University of Medical Science (approval no. 26-28). Informed consent was obtained from all subjects both verbally and in writing.

Clinical parameters. The following anthropometric measurements were obtained on admission: height $(\mathrm{cm})$, body weight $(\mathrm{BW} ; \mathrm{kg})$, BMI $\left(\mathrm{kg} / \mathrm{m}^{2}\right)$, percent ideal $\mathrm{BW}$ (\%IBW), percent triceps skin fold thickness [\%TSC; 100xTSF/reference value in the Japanese Anthropometric Reference Data (JARD) 2001 (32)] and percent arm muscle circumference [\%AMC; 100xAMC/reference value in the JARD 2001 (32)]. The SGA (22) and PG-SGA (23) were used as nutrition screening tools. The SGA rating A was regarded as well-nourished status; both $\mathrm{B}$ and $\mathrm{C}$ were regarded as malnourished status (B: Moderate; $C$ : Severe). Given that PG-SGA score $\geq 4$ is a requirement for nutrition intervention, patients were divided into two groups using a PG-SGA cutoff score of 4. Bioimpedance analysis was performed to determine FFM (kg), \%FFM, body fat mass (FAT; kg), and \%FAT using a body composition analyzer (MLT-550N; SK Medical Electronics Co.,Ltd.). Blood biochemistry tests were performed to determine the levels of total protein $(\mathrm{g} / \mathrm{dl})$, albumin $(\mathrm{g} / \mathrm{dl})$, C-reactive protein (CRP; mg/dl), serum IL-6 (pg/ml), serum TNF- $\alpha$ (pg/ml), leptin ( $\mathrm{ng} / \mathrm{ml})$, active ghrelin ( $\mathrm{fmol} / \mathrm{ml})$, and inactive (des-acryl) ghrelin (fmol/ml).

Energy metabolism. BEE was estimated using the Harris-Benedict equation (33). REE, carbohydrate oxidation, fat oxidation, and respiratory quotient (RQ) were measured using indirect calorimetry (Aeromonitor ${ }^{\circledR}$ AE310S, Minato Medical Science Co., Ltd.). REE was calculated using the Weir equation without use of urinary nitrogen (34). RQ was calculated as $\mathrm{RQ}=\mathrm{VCO}_{2} / \mathrm{VO}_{2}$. Indirect calorimetry was performed on fasted patients in the morning after resting in the supine position on a bed for $30 \mathrm{~min}$. The measurements took $10 \mathrm{~min}(35-37)$.

Energy intake. Mean daily energy intake, calculated based on daily energy intake on 3 hospital days, was used as energy intake in principle. Food intake rate (energy intake/energy provided in hospital food) and energy satisfaction rate (energy intake/energy requirement) were calculated. Energy requirement was estimated by multiplying REE by a physical activity coefficient. Because all patients were ambulant, a physical activity coefficient of 1.3 was used for all patients.

Statistical analysis. Statistical analysis was performed using statistical software SPSS version 25 (IBM, Corp.). Results are expressed as the mean \pm standard deviation. Associations between independent groups were analyzed with the $\chi^{2}$ test, the Student's t-test, or the Mann-Whitney U test as appropriate. The Kruskal-Wallis test followed by Dunn's post hoc test was used when comparing three or more groups. The 
Table I. Clinical parameters by cancer stage.

\begin{tabular}{|c|c|c|c|}
\hline Characteristics & Stage I, II, n=27 & Stage III, IV, n=24 & P-value \\
\hline Male/female & $21 / 6$ & $17 / 7$ & $0.570^{\mathrm{a}}$ \\
\hline Age, years & $64 \pm 7$ & $64 \pm 11$ & $0.571^{\mathrm{b}}$ \\
\hline Cancer stage, I/II/III/IV & $16 / 11 / 0 / 0$ & $0 / 0 / 13 / 11$ & \\
\hline Cancer origin, esophageal/gastric/colorectal & $7 / 11 / 9$ & $10 / 4 / 10$ & $0.158^{\mathrm{a}}$ \\
\hline \multicolumn{4}{|l|}{ Anthropometrics } \\
\hline Height, m & $1.67 \pm 0.09$ & $1.61 \pm 0.10$ & $0.054^{\mathrm{c}}$ \\
\hline $\mathrm{BW}, \mathrm{kg}$ & $63.6 \pm 11.1$ & $53.6 \pm 10.4$ & $<0.01^{\mathrm{c}}$ \\
\hline Body mass index, $\mathrm{kg} / \mathrm{m}^{2}$ & $22.8 \pm 3.0$ & $20.5 \pm 3.5$ & $<0.05^{\mathrm{c}}$ \\
\hline Body fat mass, $\mathrm{kg}$ & $15.2 \pm 7.2$ & $13.0 \pm 6.4$ & $0.295^{\mathrm{c}}$ \\
\hline Fat-free mass, $\mathrm{kg}(\mathrm{n}=46)$ & $47.7 \pm 9.0$ & $41.0 \pm 9.1$ & $<\mathbf{0 . 0 5}$ \\
\hline$\%$ TSF $(n=49)$ & $101.5 \pm 38.0$ & $69.9 \pm 30.6$ & $<0.01^{\mathrm{c}}$ \\
\hline$\% \operatorname{AMC}(\mathrm{n}=49)$ & $102.6 \pm 12.3$ & $96.9 \pm 13.6$ & $0.131^{\mathrm{c}}$ \\
\hline \multicolumn{4}{|l|}{ Nutritional assessment } \\
\hline SGA, well-nourished/malnourished ${ }^{\mathrm{d}}$ & $22 / 5$ & $9 / 15$ & $<0.01^{\text {a }}$ \\
\hline PG-SGA, $<4 / \geq 4(n=49)$ & $13 / 13$ & $3 / 20$ & $<0.01^{\mathrm{a}}$ \\
\hline BW loss in 6 months, $\%$ & $1.7 \pm 3.5$ & $6.3 \pm 7.4$ & $<\mathbf{0 . 0 5}^{\mathrm{b}}$ \\
\hline Food intake rate, $\%(n=42)$ & $99 \pm 3$ & $76 \pm 33$ & $<0.01^{b}$ \\
\hline \multicolumn{4}{|l|}{ Energy metabolism } \\
\hline BEE, kcal/day & $1,348 \pm 182$ & $1,189 \pm 187$ & $<0.01^{\mathrm{c}}$ \\
\hline REE, kcal/day & $1,371 \pm 193$ & $1,260 \pm 265$ & $0.091^{\mathrm{c}}$ \\
\hline $\mathrm{REE} / \mathrm{BEE}$ & $1.02 \pm 0.09$ & $1.06 \pm 0.12$ & $0.214^{\mathrm{c}}$ \\
\hline $\mathrm{REE} / \mathrm{BW}, \mathrm{kcal} / \mathrm{kg} / \mathrm{day}$ & $22.0 \pm 2.3$ & $23.8 \pm 3.9$ & $0.086^{\mathrm{c}}$ \\
\hline BEE/FFM, kcal/kg/day $(\mathrm{n}=46)$ & $29.1 \pm 3.9$ & $31.5 \pm 5.7$ & $0.095^{\mathrm{c}}$ \\
\hline $\mathrm{RQ}$ & $0.82 \pm 0.10$ & $0.80 \pm 0.08$ & $0.507^{\mathrm{c}}$ \\
\hline \multicolumn{4}{|l|}{ Blood biochemistry } \\
\hline Total protein, g/dl & $6.8 \pm 0.5$ & $6.7 \pm 0.5$ & $0.302^{c}$ \\
\hline Albumin, g/dl & $4.1 \pm 0.4$ & $3.6 \pm 0.4$ & $<0.01^{\mathrm{c}}$ \\
\hline C-reactive protein, mg/dl (n=49) & $0.2 \pm 0.2$ & $1.4 \pm 2.1$ & $<0.01^{b}$ \\
\hline IL-6, pg/ml $(\mathrm{n}=49)$ & $1.9 \pm 1.2$ & $6.6 \pm 7.3$ & $<0.01^{b}$ \\
\hline TNF- $\alpha, p g / m l(n=49)$ & $0.9 \pm 0.4$ & $1.4 \pm 0.6$ & $<0.01^{\mathrm{c}}$ \\
\hline Leptin, $\mathrm{ng} / \mathrm{ml}(\mathrm{n}=48)$ & $7.4 \pm 6.8$ & $8.5 \pm 12.5$ & $0.691^{\mathrm{c}}$ \\
\hline Active ghrelin, fmol/ml ( $\mathrm{n}=49)$ & $11.8 \pm 9.4$ & $14.9 \pm 15.6$ & $0.398^{\mathrm{c}}$ \\
\hline Des-acyl ghrelin, fmol/ml (n=49) & $146.0 \pm 121.6$ & $133.6 \pm 68.4$ & $0.489^{\mathrm{b}}$ \\
\hline
\end{tabular}

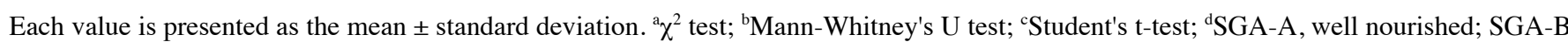
or SGA-C, malnourished. Bold numbers indicate statistically significant results. BW, body weight; TSF, triceps skinfold thickness; AMC, arm muscle circumference; SGA, Subjective Global Assessment; PG-SGA, Patient-Generated Subjective Global Assessment; BEE, basal energy expenditure; REE, resting energy expenditure; RQ, respiratory quotient; IL, interleukin; TNF, tumor necrosis factor.

Jonckheere-Terpstra trend test was used to examine trends. For correlation analysis, the Spearman's rank correlation coefficient was used. $\mathrm{P}<0.05$ was used to indicate a statistically significant difference.

\section{Results}

Patient characteristics. Patient characteristics were summarized by cancer stage and by cancer location (Tables I and II, respectively). Subjects were 51 patients (38 men, 13 women) aged $<80$ years admitted for treatment of diagnosed gastrointestinal cancer. The distribution of cancer stages I, II, III, and IV was $16,11,13$, and 11 patients, respectively. As for cancer location, the number of patients with esophageal, gastric, and colorectal cancer was 17, 15, and 19 , respectively.

Nutritional screening. All 51 patients were assessed using the SGA, and 49 were assessed using the PG-SGA. The SGA identified more well-nourished patients in stages I/II than in stages III/IV $(\mathrm{P}<0.01)$, with more malnourished patients in stages III/IV than in stages I/II $(\mathrm{P}<0.01)$. Also, the PG-SGA identified more patients requiring nutrition intervention in stages III/IV than in stages I/II $(\mathrm{P}<0.01)$. The proportion of 
Table II. Clinical parameters by cancer location.

\begin{tabular}{|c|c|c|c|c|}
\hline Characteristics & Esophageal $(n=17)$ & Gastric $(n=15)$ & Colorectal $(n=19)$ & P-value \\
\hline Male/female & $16 / 1$ & $11 / 4$ & $11 / 8$ & $<\mathbf{0 . 0 5}^{\mathrm{b}}$ \\
\hline Age, years & $65 \pm 9$ & $65 \pm 8$ & $63 \pm 0$ & $0.844^{\mathrm{c}}$ \\
\hline Cancer stage, I/II/III/IV & $6 / 1 / 6 / 4$ & $7 / 4 / 1 / 3$ & $3 / 6 / 6 / 4$ & $0.189^{\mathrm{b}}$ \\
\hline \multicolumn{5}{|l|}{ Anthropometrics } \\
\hline Height, m & $1.67 \pm 0.08$ & $1.63 \pm 0.11$ & $1.63 \pm 0.10$ & $0.600^{c}$ \\
\hline $\mathrm{BW}, \mathrm{kg}$ & $57.0 \pm 11.2$ & $61.6 \pm 14.8$ & $58.5 \pm 9.8$ & $0.387^{\mathrm{c}}$ \\
\hline Body mass index, $\mathrm{kg} / \mathrm{m}^{2}$ & $20.4 \pm 3.0$ & $22.7 \pm 3.3$ & $22.2 \pm 3.6$ & $0.161^{\mathrm{c}}$ \\
\hline Body fat mass, $\mathrm{kg}$ & $11.4 \pm 5.5$ & $17.3 \pm 6.6$ & $14.2 \pm 7.4$ & $0.565^{\mathrm{c}}$ \\
\hline Fat-free mass, $\mathrm{kg}(\mathrm{n}=46)$ & $45.6 \pm 10.1$ & $43.8 \pm 10.8$ & $43.9 \pm 8.4$ & $0.943^{c}$ \\
\hline$\% \operatorname{TSF}(n=49)$ & $68.1 \pm 30.7$ & $108.5 \pm 44.7$ & $86.3 \pm 30.5$ & $0.048^{c}$ \\
\hline$\%$ AMC $(n=49)$ & $97.2 \pm 10.9$ & $99.2 \pm 12.4$ & $102.6 \pm 15.3$ & $0.643^{c}$ \\
\hline \multicolumn{5}{|l|}{ Nutritional assessment } \\
\hline SGA, well-nourished/malnourished ${ }^{\mathrm{a}}$ & $9 / 8$ & $10 / 5$ & $12 / 7$ & $0.704^{b}$ \\
\hline PG-SGA, $<4 / \geq 4(n=49)$ & $6 / 10$ & $6 / 8$ & $4 / 15$ & $0.368^{\mathrm{b}}$ \\
\hline BW loss in 6 months, $\%$ & $4.2 \pm 6.3$ & $3.5 \pm 6.8$ & $3.9 \pm 5.6$ & $0.910^{c}$ \\
\hline Food intake rate, $\%(n=42)$ & $79 \pm 29$ & $90 \pm 25$ & $74 \pm 39$ & $0.077^{\mathrm{c}}$ \\
\hline \multicolumn{5}{|l|}{ Energy metabolism } \\
\hline BEE, kcal/day & $1,249 \pm 198$ & $1,312 \pm 234$ & $1,265 \pm 175$ & $0.500^{\mathrm{c}}$ \\
\hline REE, kcal/day & $1,311 \pm 222$ & $1,319 \pm 227$ & $1,324 \pm 262$ & $0.892^{c}$ \\
\hline REE/BEE & $1.05 \pm 0.10$ & $1.01 \pm 0.07$ & $1.05 \pm 0.13$ & $0.462^{\mathrm{c}}$ \\
\hline $\mathrm{REE} / \mathrm{BW}, \mathrm{kcal} / \mathrm{kg} / \mathrm{day}$ & $23.5 \pm 2.4$ & $21.9 \pm 3.2$ & $22.8 \pm 3.9$ & $0.091^{\mathrm{c}}$ \\
\hline REE/FFM, kcal/kg/day $(\mathrm{n}=46)$ & $29.0 \pm 3.4$ & $30.7 \pm 4.8$ & $31.0 \pm 6.2$ & $0.624^{\mathrm{c}}$ \\
\hline RQ & $0.81 \pm 0.07$ & $0.80 \pm 0.07$ & $0.83 \pm 0.12$ & $0.823^{\mathrm{c}}$ \\
\hline \multicolumn{5}{|l|}{ Blood biochemistry } \\
\hline Total protein, g/dl & $6.8 \pm 0.6$ & $6.9 \pm 0.5$ & $6.6 \pm 0.5$ & $0.225^{\mathrm{c}}$ \\
\hline Albumin, g/dl & $3.9 \pm 0.5$ & $4.0 \pm 0.5$ & $3.8 \pm 0.3$ & $0.229^{c}$ \\
\hline C-reactive protein, mg/dl $(\mathrm{n}=49)$ & $0.9 \pm 1.2$ & $1.2 \pm 2.7$ & $0.4 \pm 0.7$ & $0.522^{c}$ \\
\hline IL-6, pg/ml $(\mathrm{n}=49)$ & $4.4 \pm 4.5$ & $4.2 \pm 6.7$ & $3.8 \pm 5.7$ & $0.723^{c}$ \\
\hline TNF- $\alpha, p g / m l(n=49)$ & $1.0 \pm 0.5$ & $1.1 \pm 0.6$ & $1.2 \pm 0.6$ & $0.688^{c}$ \\
\hline Leptin, ng/ml (n=48) & $5.5 \pm 2.6$ & $6.7 \pm 3.9$ & $11.3 \pm 15.4$ & $0.792^{c}$ \\
\hline Active ghrelin, fmol/ml ( $\mathrm{n}=49)$ & $15.2 \pm 10.2$ & $14.2 \pm 19.2$ & $10.8 \pm 6.9$ & $0.310^{c}$ \\
\hline Des-acyl ghrelin, fmol/ml (n=49) & $154.8 \pm 99.4$ & $110.4 \pm 89.3$ & $153.1 \pm 111.0$ & $0.053^{\mathrm{c}}$ \\
\hline
\end{tabular}

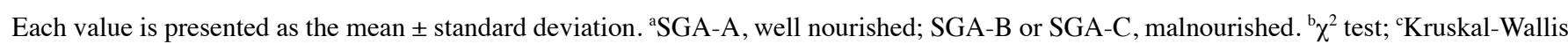
analysis. Bold numbers indicate statistically significant results. BW, body weight; TSF, triceps skinfold thickness; AMC, arm muscle circumference; SGA, Subjective Global Assessment; PG-SGA, Patient-Generated Subjective Global Assessment; BEE, basal energy expenditure; REE, resting energy expenditure; RQ, respiratory quotient; IL, interleukin; TNF, tumor necrosis factor.

patients with malnourished status increased with cancer stage progression (Table I).

When the SGA results were examined by cancer location, the numbers of well-nourished patients and malnourished patients were similar in esophageal cancer, whereas there were more well-nourished patients than malnourished patients in gastric cancer and colorectal cancer. The PG-SGA identified more patients requiring nutritional intervention than not requiring nutritional intervention, irrespective of cancer location. However, nutritional screening results using both the SGA and PG-SGA showed no significant association with cancer location. There was no significant difference between cancer locations, although \%TSF was lower in esophageal cancer than colorectal cancer (Dunn's post hoc analysis, $\mathrm{P}=0.043$, Table II).

Anthropometric measurements, body composition analysis, energy intake. BW, BMI, \% IBW, and FFM were significantly lower in stages III/IV than stages I/II. There was no significant difference in \%FAT, but FAT decreased as cancer stage progressed $(15.2 \pm 7.2 \mathrm{~kg}$ in stages I/II vs. $13.0 \pm 6.4 \mathrm{~kg}$ in stages III/IV). Also, \%TSF was lower in stages III/IV (69.9\%) than in stages I/II (101.5\%). Percent BW loss in 6 months was significantly larger in stages III/IV $(6.3 \pm 7.4 \%)$ than in stages I/II $(1.7 \pm 3.5 \%)$. Food intake rate was significantly lower in stages III/IV $(76 \pm 33 \%)$ than in stages I/II $(99 \pm 3 \%)$ (Table I). 

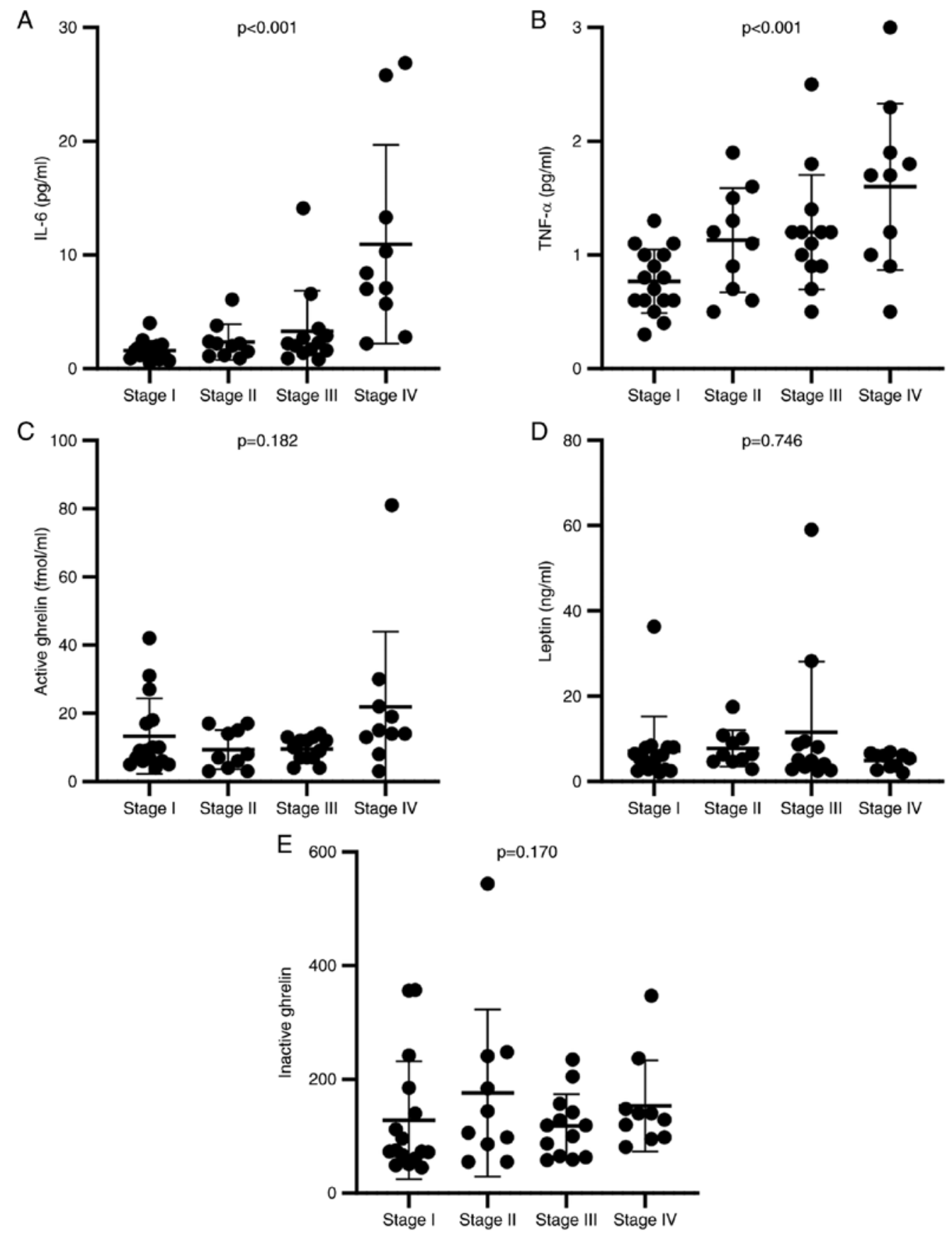

Figure 1. Cancer stage and levels of inflammatory cytokines and appetite-regulating hormones. Association of cancer stage with (A) IL-6, (B) TNF- $\alpha$, (C) active ghrelin, (D) leptin and (E) inactive ghrelin. Jonckheere-Terpstra trend test was performed to analyze the data. IL, interleukin; TNF, tumor necrosis factor.

Blood biochemistry. Albumin level tended to be lower while CRP level tended to be higher in stages III/IV than in stages I/II. Levels of inflammatory cytokines, such as IL-6 and TNF- $\alpha$ were significantly higher in stages III/IV than in stages I/II. The trend test of inflammatory cytokines showed significant increases with cancer stage progression (Fig. 1A and B). Also, the levels of the appetite-regulating hormones active ghrelin and leptin were not associated with cancer stage (Fig. 1C-E). However, the level of active ghrelin was significantly increased in stage IV compared with stage III (Mann-Whitney's U test, $\mathrm{P}=0.009)$.

Energy metabolism. The trend test of BEE and REE showed significant decreases with cancer stage progression
(Fig. 2A and B). Similarly, BW and FFM showed significant decreases with cancer stage progression (Fig. 2C and D). Indices of energy metabolism, such as REE/BW and REE/FFM, tended to become higher with cancer stage progression (Fig. 2E and F). Results of this study revealed an energy requirement per $\mathrm{BW}$ of $22 \mathrm{kcal}$ and a stress coefficient of 1.0 for patients with stages I/II disease and an energy requirement per BW of $24 \mathrm{kcal}$ and a stress coefficient of 1.1 for patients with stages III/IV disease (stress coefficient was calculated using $\mathrm{REE} / \mathrm{BEE}$ ).

Association of inflammatory cytokines with FFM and energy metabolism. Inflammatory cytokines mediate cancer progression, and they either decrease FFM or increase energy 

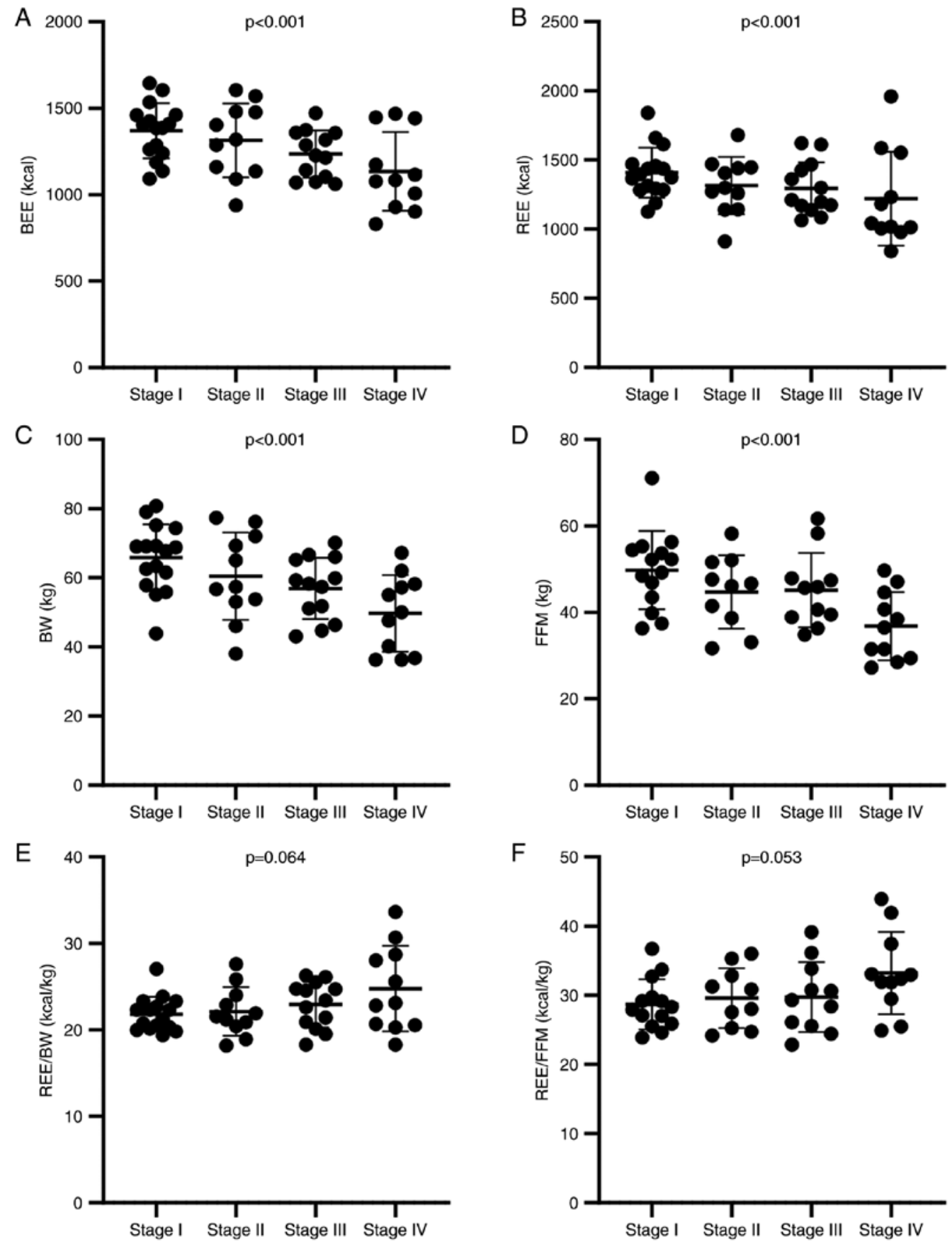

Figure 2. Energy expenditure by cancer stage. Association of cancer stage with (A) BEE, (B) REE, (C) BW, (D) FFM, (E) REE/BW and (F) REE/FFM Jonckheere-Terpstra trend test was performed to analyze the data. BEE, basal energy expenditure; REE, resting energy expenditure; BW, body weight; FFM, fat-free mass.

metabolism. Therefore, their correlations were analyzed. FFM and inflammatory cytokines were found to have a negative correlation (Fig. 3A and B).

REE/FFM was used as an energy metabolism index and showed a positive but insignificant correlation with both IL-6 and TNF- $\alpha$ (Fig. 3C and D). On the other hand, food intake rate significantly decreased as the levels of inflammatory cytokines increased (Fig. 3E and F).

Association of appetite-regulating hormones with FFM and energy metabolism. As shown in Fig. 1C, the level of active ghrelin was increased in patients with stage IV cancer. This elevated level could have a compensatory function maintain homeostasis. Therefore, the correlations related to appetite-regulating hormones are presented separately in Figs. 4 and 5 for stages I/II and stages III/IV, respectively.

The increased level of active ghrelin was correlated with a significant increase in REE/FFM and a significant decrease in food intake rate (Fig. 4B and E). On the other hand, an increased level of leptin tended to be associated with mild increases in FFM and food intake rate (Fig. 4B and F).

Fig. 5 also shows the correlations between the inflammatory cytokines and appetite-regulating hormones. There was 

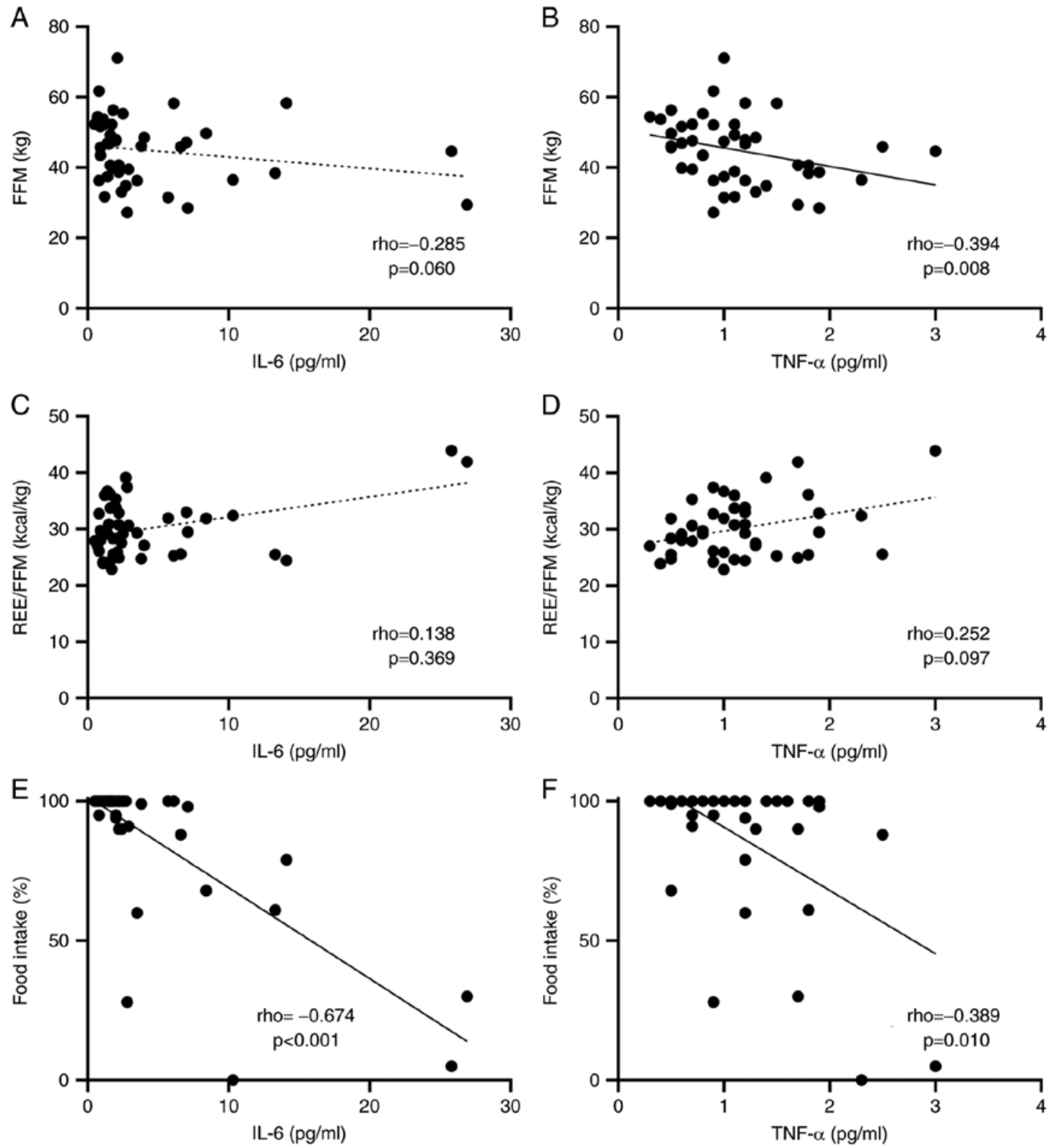

Figure 3. Correlations of the levels of inflammatory cytokines with FFM, energy metabolism and food intake. Correlations of FFM with (A) IL-6 and (B) TNF- $\alpha$. Correlations of REE/FFM with (C) IL-6 and (D) TNF- $\alpha$. Correlations of food intake rate with (E) IL-6 and (F) TNF- $\alpha$. Rho indicates Spearman's rank correlation coefficient. P-values and rho values on each graph were calculated for all patients. FFM, fat-free mass; IL, interleukin; TNF, tumor necrosis factor; REE, resting energy expenditure.

a significant positive correlation between the level of active ghrelin and IL-6 (Fig. 5A).

\section{Discussion}

This study confirmed increases in the inflammatory cytokine levels with cancer stage progression and suggests the possible correlation of increases in inflammatory cytokine levels with an increase in energy metabolism and decreases in food intake rate and FFM. There were also correlations of the level of active ghrelin with the level of IL-6 and energy metabolism in cancer patients.

Nutritional status in cancer patients was assessed using two screening tools, the SGA and PG-SGA. Regardless of the tool used, increased numbers of malnourished patients were observed with cancer stage progression. Also, the PG-SGA identified more patients requiring nutritional intervention than the SGA did. In particular, patients requiring nutritional intervention accounted for $79 \%$ of patients with colorectal cancer. The sensitivity of the PG-SGA appeared to be higher than that of the SGA because the PG-SGA, but not the SGA, includes patient concerns in the assessment.

This study confirmed increases in inflammatory cytokine levels in blood with cancer stage progression. IL-6 tended to be particularly high in patients with stage IV cancer, while TNF- $\alpha$ increased stepwise as cancer stage progressed. The proportion of patients with stage IV cancer was relatively low, which could explain the non-significant correlations with FFM and energy metabolism. It is noteworthy that both cytokines showed negative correlations with food intake rate. 

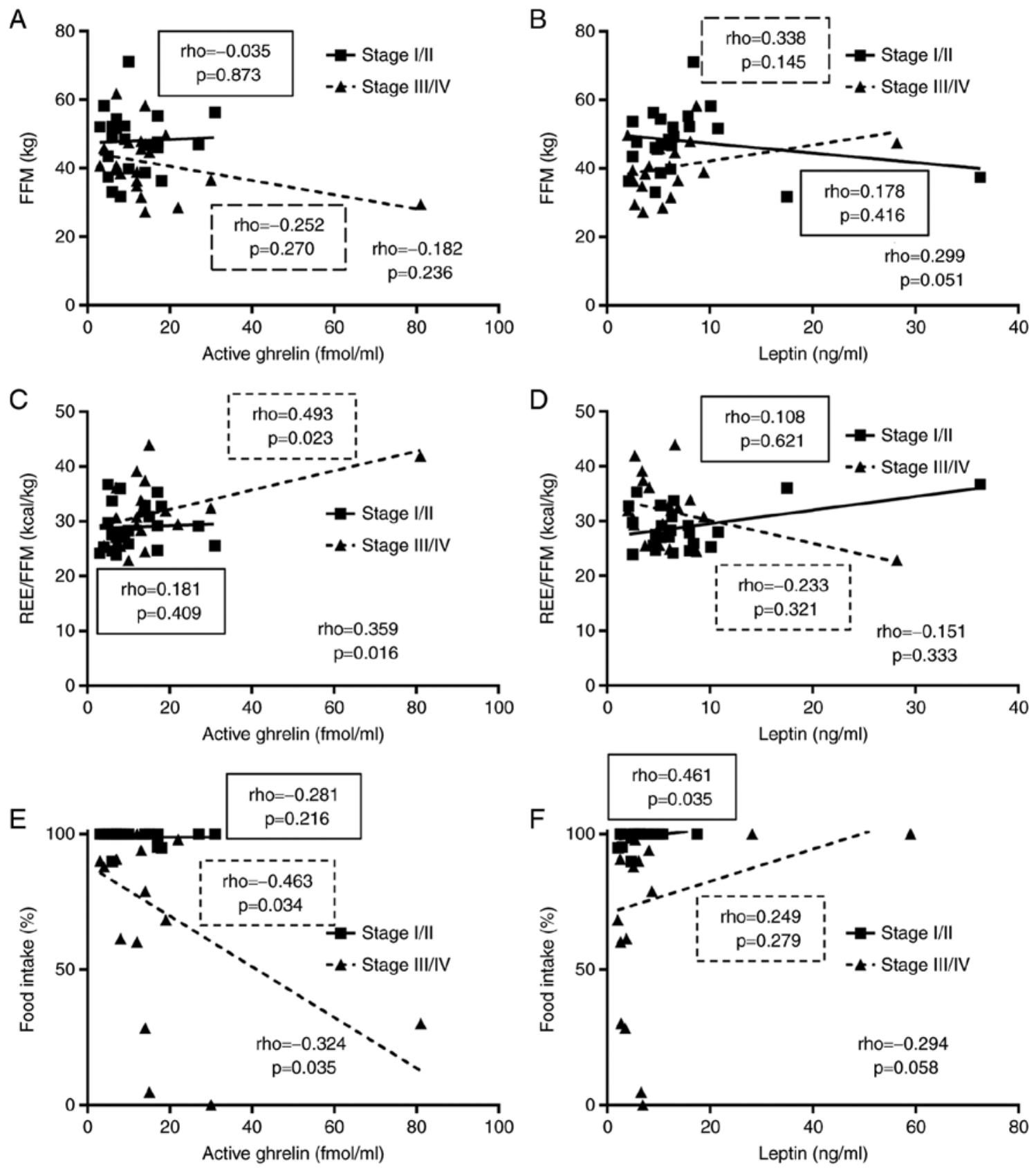

Figure 4. Correlations of the levels of appetite-regulating hormones with FFM, energy metabolism and food intake. Correlations of FFM with (A) active ghrelin and (B) leptin. Correlations of REE/FFM with (C) active ghrelin and (D) leptin. Correlations of food intake rate with (E) active ghrelin and (F) leptin. Rho indicates Spearman's rank correlation coefficient. P-values and rho values on each graph were calculated for all patients. The rho and P-values for patients with stage I/II and III/IV cancer are enclosed in solid and dotted boxes, respectively, and those for all patients are not enclosed. FFM, fat-free mass; REE, resting energy expenditure.

Taken together, the levels of inflammatory cytokines increase with cancer progression, and this leads to decreases in food intake rate, increased energy metabolism, and decreased FFM.

Ghrelin is known to suppress energy metabolism and inflammatory cytokines, such as IL-6 and TNF- $\alpha$ (15). However, in this study, energy metabolism and IL-6 were positively correlated with active ghrelin. A state of ghrelin resistance exists in cancer patients. Therefore, energy metabolism was not suppressed even at high active ghrelin levels, and IL-6 appeared to correlate with active ghrelin. In line with this, food intake rate was also negatively correlated with active ghrelin in cancer patients, suggesting that the increased ghrelin failed to compensate for increased appetite.

It has been speculated that the level of serum leptin decreases with cancer progression because it is a hormone released from fat cells (38) and is known to suppress appetite (39). However, this study did not find any significant differences among cancer stages or among cancer locations.

This study has some limitations. First, this is a single-center study, and therefore generalization of the results is limited. Second, data were collected before treatment, and changes in energy metabolism with time were not examined. Third, the 

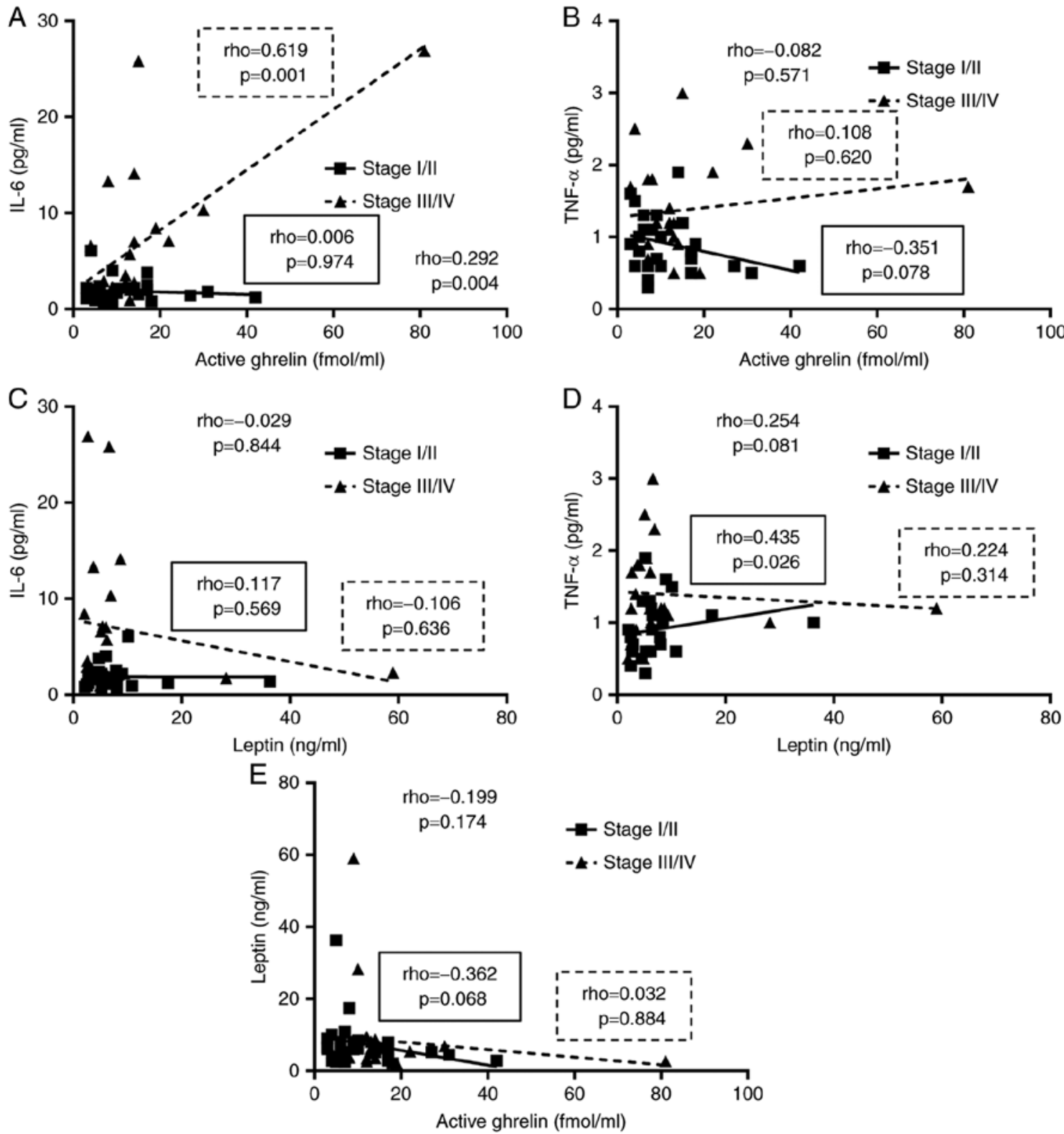

Figure 5. Correlations of the levels of inflammatory cytokines with appetite-regulating hormones. Correlations of active ghrelin with (A) IL-6 and (B) TNF- $\alpha$. Correlations of leptin with (C) IL-6, (D) TNF- $\alpha$ and (E) active ghrelin. Rho indicates Spearman's rank correlation coefficient. P-values and rho values on each graph were calculated for all patients. The rho and P-values for patients with stage I/II and III/IV cancer are enclosed in solid and dotted boxes, respectively, and those for all patients are not enclosed. IL, interleukin; TNF, tumor necrosis factor.

imbalances in patient sex, cancer stage, and cancer location might have influenced the results. More accurate findings can be obtained by using a larger sample size with adjustments for patient sex, cancer stage, and cancer location.

In conclusion, analysis by cancer stage in patients with gastrointestinal cancer showed that levels of inflammatory cytokines increased with cancer stage progression, which may lead to decreases in food intake rate and FFM, and with increases in energy expenditure. In particular, the level of IL-6 influenced energy metabolism. Furthermore, the TNF- $\alpha$ level was significantly associated with decreases in FFM. In terms of the appetite-regulating hormones, we found that the level of active ghrelin was positively correlated with that of IL-6 and with energy metabolism, which suggests a state of ghrelin resistance.

\section{Acknowledgements}

Not applicable.

\section{Funding}

The present study was supported in part by a Grant-in-Aid for Scientific Research from The Ministry of Education, Culture, Sports, Science and Technology of Japan (grant no. 18K10990 to $\mathrm{SB})$.

\section{Availability of data and materials}

The datasets used and analyzed during the present study are available from the corresponding author on reasonable request. 


\section{Authors' contributions}

ASh, SB and MS conceived the study design. ASh and SB performed data analysis. ASh, MKu, HM, ASo, OI, AA, KT, MKo, HI, MT and MS performed the acquisition and interpretation of the data. ASh, SB and MS wrote the manuscript. $\mathrm{MKu}, \mathrm{HM}$, ASo, OI, AA, KT, MKo, HI and MT revised and edited the manuscript. MKu, HM, ASo, OI, KT, MKo and HI treated the patients presented in the manuscript. AA, MT and MS supervised the study. All authors read and approved the final manuscript.

\section{Ethics approval and consent to participate}

The present study was conducted with approval by The Ethics Committee of Shiga University of Medical Science (approval no. 26-28). Informed consent was obtained from all subjects both verbally and in writing.

\section{Patient consent for publication}

Not applicable.

\section{Competing interests}

The authors declare that they have no competing interests.

\section{References}

1. Arends J, Baracos V, Bertz H, Bozzetti F, Calder PC, Deutz NEP, Erickson N, Laviano A, Lisanti MP, Lobo DN, et al: ESPEN expert group recommendations for action against cancer-related malnutrition. Clin Nutr 36: 1187-1196, 2017.

2. Zhang L, Lu Y and Fang Y: Nutritional status and related factors of patients with advanced gastrointestinal cancer. Br J Nutr 111: 1239-1244, 2014.

3. Grivennikov SI and Karin M: Inflammatory cytokines in cancer: Tumour necrosis factor and interleukin 6 take the stage. Ann Rheum Dis 70 (Suppl 1): i104-i108, 2011.

4. Ashizawa T, Okada R, Suzuki Y, Takagi M, Yamazaki T, Sumi T, Aoki T, Ohnuma S and Aoki T: Clinical significance of interleukin-6 (IL-6) in the spread of gastric cancer: Role of IL-6 as a prognostic factor. Gastric Cancer 8: 124-131, 2005.

5. Guthrie GJ, Roxburgh CS, Horgan PG and McMillan DC: Does interleukin-6 link explain the link between tumour necrosis local and systemic inflammatory responses and outcome in patients with colorectal cancer? Cancer Treat Rev 39: 89-96, 2013.

6. Shimazaki J, Goto Y, Nishida K, Tabuchi T, Motohashi G and Ubukata $\mathrm{H}$ : In patients with colorectal cancer, preoperative serum interleukin-6 level and granulocyte/lymphocyte ratio are clinically relevant biomarkers of long-term cancer progression. Oncology 84: 356-361, 2013.

7. Ma Y, Ren Y, Dai ZJ, Wu CJ, Ji YH and Xu J: IL-6, IL-8 and TNF- $\alpha$ levels correlate with disease stage in breast cancer patients. Adv Clin Exp Med 26: 421-426, 2017.

8. Fearon K, Strasser F, Anker SD, Bosaeus I, Bruera E, Fainsinger RL, Jatoi A, Loprinzi C, MacDonald N, Mantovani G, et al: Definition and classification of cancer cachexia: An international consensus. Lancet Oncol 12: 489-495, 2011.

9. Braun TP and Marks DL: Pathophysiology and treatment of inflammatory anorexia in chronic disease. J Cachexia Sarcopenia Muscle 1: 135-145, 2010.

10. Li YP and Reid MB: NF-kappaB mediates the protein loss induced by TNF-alpha in differentiated skeletal muscle myotubes. Am J Physiol Regul Integr Comp Physiol 279: R1165-R1170, 2000.

11. Kojima M, Hosoda H, Date Y, Nakazato M, Matsuo H and Kangawa K: Ghrelin is a growth-hormone-releasing acylated peptide from stomach. Nature 402: 656-660, 1999.
12. Mihalache L, Gherasim A, Nita O, Ungureanu MC, Pădureanu SS, Gavril RS and Arhire LI: Effects of ghrelin in energy balance and body weight homeostasis. Hormones (Athens) 15: 186-196, 2016.

13. Cui H, Lopez M and Rahmouni K: The cellular and molecular bases of leptin and ghrelin resistance in obesity. Nat Rev Endocrinol 13: 338-351, 2017

14. Kirchner H, Gutierrez JA, Solenberg PJ, Pfluger PT, Czyzyk TA, Willency JA, Schürmann A, Joost HG, Jandacek RJ, Hale JE, et al: GOAT links dietary lipids with the endocrine control of energy balance. Nat Med 15: 741-745, 2009.

15. Suzuki H, Asakawa A, Amitani H, Nakamura $\mathrm{N}$ and Inui A: Cancer cachexia-pathophysiology and management. J Gastroenterol 48: 574-594, 2013.

16. Nakazato M, Murakami N, Date Y, Kojima M, Matsuo H, Kangawa $\mathrm{K}$ and Matsukura S: A role for ghrelin in the central regulation of feeding. Nature 409: 194-198, 2001.

17. Shimizu $Y$, Nagaya N, Isobe T, Imazu M, Okumura $H$, Hosoda H, Kojima M, Kangawa K and Kohno N: Increased plasma ghrelin level in lung cancer cachexia. Clin Cancer Res 9: 774-778, 2003.

18. Wolf I, Sadetzki S, Kanety H, Kundel Y, Pariente C, Epstein N, Oberman B, Catane R, Kaufman B and Shimon I: Adiponectin, ghrelin, and leptin in cancer cachexia in breast and colon cancer patients. Cancer 106: 966-973, 2006

19. Kerem M, Ferahkose Z, Yilmaz UT, Pasaoglu H, Ofluoglu E, Bedirli A, Salman B, Sahin TT and Akin M: Adipokines and ghrelin in gastric cancer cachexia. World J Gastroenterol 14: 3633-3641, 2008.

20. Garcia JM, Garcia-Touza M, Hijazi RA, Taffet G, Epner D, Mann D, Smith RG, Cunningham GR and Marcelli M: Active ghrelin levels and active to total ghrelin ratio in cancer-induced cachexia. J Clin Endocrinol Metab 90: 2920-2926, 2005.

21. Nagaya N, Uematsu M, Kojima M, Date Y, Nakazato M, Okumura H, Hosoda H, Shimizu W, Yamagishi M, Oya H, et al: Elevated circulating level of ghrelin in cachexia associated with chronic heart failure: Relationships between ghrelin and anabolic/catabolic factors. Circulation 104: 2034-2038, 2001.

22. Baker JP, Detsky AS, Wesson DE, Wolman SL, Stewart S, Whitewell J, Langer B and Jeejeebhoy KN: Nutritional assessment: A comparison of clinical judgement and objective measurements. N Engl J Med 306: 969-972, 1982.

23. Isenring EA, Capra S and Bauer JD: Nutrition intervention is beneficial in oncology outpatients receiving radiotherapy to the gastrointestinal or head and neck area. Br J Cancer 91: 447-452, 2004.

24. Bauer J, Capra S and Ferguson M: Use of the scored Patient-Generated Subjective Global Assessment (PG-SGA) as a nutrition assessment tool in patients with cancer. Eur J Clin Nutr 56: 779-785, 2002.

25. Dempsey DT, Feurer ID, Knox LS, Crosby LO, Buzby GP and Mullen JL: Energy expenditure in malnourished gastrointestinal cancer patients. Cancer 53: 1265-1273, 1984.

26. Hansell DT, Davies JW and Burns HJ: The relationship between resting energy expenditure and weight loss in benign and malignant disease. Ann Surg 203: 240-245, 1986.

27. Bosaeus I, Daneryd P, Svanberg E and Lundholm K: Dietary intake and resting energy expenditure in relation to weight loss in unselected cancer patients. Int J Cancer 93: 380-383, 2001.

28. Moses AW, Slater C, Preston T, Barber MD and Fearon KC: Reduced total energy expenditure and physical activity in cachectic patients with pancreatic cancer can be modulated by an energy and protein dense oral supplement enriched with n-3 fatty acids. Br J Cancer 90: 996-1002, 2004.

29. Staal-van den Brekel AJ, Schols AM, ten Velde GP, Buurman WA and Wouters EF: Analysis of the energy balance in lung cancer patients. Cancer Res 54: 6430-6433, 1994.

30. Falconer JS, Fearon KC, Plester CE, Ross JA and Carter DC: Cytokines, the acute-phase response, and resting energy expenditure in cachectic patients with pancreatic cancer. Ann Surg 219: 325-331, 1994.

31. Omagari K, Iwami H, Kaji M, Ishii Y, Matsutake S, Ichimura M, Kato S, Takeshhita, Ichikawa $\mathrm{T}$ and Nakao K: The relationship between energy expenditure and type or stage of cancer. Acta Medica Nagasakiensia 57: 33-40, 2012.

32. MoriwakiH, Aoyagi S, Ishizuka Y, et al: Japanese Anthropometric Reference Data 2001. JARD 2001. Jpn Nutr Assess 19: 45-81, 2002 . 
33. Harris JA and Benedict FG: A biometric study of human basal metabolism. Proc Natl Acad Sci USA 4: 370-373, 1918.

34. Weir JB: New methods for calculating metabolic rate with special reference to protein metabolism. J Physiol 109: 1-9, 1949.

35. Sasaki M, Johtatsu T, Kurihara M, Iwakawa H, Tanaka T, Bamba S, Tsujikawa T, Fujiyama Y and Andoh A: Energy expenditure in Japanese patients with severe or moderate ulcerative colitis. J Clin Biochem Nutr 47: 32-36, 2010.

36. Sasaki M, Okamoto H, Johtatsu T, Kurihara M, Iwakawa H, Tanaka T, Shiomi H, Naka S, Kurumi Y and Tani T: Resting energy expenditure in patients undergoing pylorus preserving pancreatoduodenectomies for bile duct cancer or pancreatic tumors. J Clin Biochem Nutr 48: 183-186, 2011.
37. Takaoka A, Sasaki M, Nakanishi N, Kurihara M, Ohi A, Bamba S and Andoh A: Nutritional screening and clinical outcome in hospitalized patients with crohn's disease. Ann Nutr Metab 71: 266-272, 2017.

38. Zhang Y, Proenca R, Maffei M, Barone M, Leopold L and Friedman JM: Positional cloning of the mouse obese gene and its human homologue. Nature 372: 425-432, 1994.

39. Satoh N, Ogawa Y, Katsuura G, Tsuji T, Masuzaki H, Hiraoka J, Okazaki T, Tamaki M, Hayase M, Yoshimasa Y, et al: Pathophysiological significance of the obese gene product, leptin, in ventromedial hypothalamus (VMH)-lesioned rats: Evidence for loss of its satiety effect in VMH-lesioned rats. Endocrinology 138: 947-954, 1997. 